\title{
Preliminary Investigation into Causal Factors for Engineering Enrollment
}

\author{
Jake Armstrong, M.A.Sc. Candidate and David S. Strong, Professor \& NSERC Chair in Design Engineering \\ Faculty of Engineering and Applied Science, Queen's University, Kingston, Ontario \\ jake.armstrong@queensu.ca; strongd@queensu.ca
}

\begin{abstract}
In Canada, the majority of university-bound students are required to choose their post-secondary program during their final year of high school. This is an important decision, and is difficult and confusing for many students with limited information to make these careerimpacting choices. Past research suggests that the engineering profession is not understood in the public domain, especially in elementary and secondary schools. To further investigate this issue, a mixed-methods study is being conducted to examine the factors behind students' selection of engineering. Self-reported retrospective satisfaction with program selection is used as an indicator for successful decision-making. Indicated factors were compared with demographics to determine if they vary in importance by gender, year or personal relationships with professional engineers. This paper discusses trends that are evolving from the first phase of processing. Extensive quantitative and qualitative analysis is still underway on the full package of data, and further findings will be published at a later date.
\end{abstract}

Keywords: Undergraduate program selection, undergraduate program transfer, program satisfaction, engineering outreach, engineering education

\section{INTRODUCTION}

In the Ontario secondary school system, students are expected to differentiate their course load as early as their first year, based on their plans for further education and/or vocation. Halfway through their final year, those wishing to directly proceed to university are asked to select their program of study. Unsurprisingly, these chosen programs are not always what the students expected. In many fields, but especially in engineering, student retention can become an issue, reported as low as $49 \%$ in some cases [1]. At Queen's University, a mid-size university in Eastern Ontario, recent analysis indicates that the engineering program has a retention rate of $89 \%$ [2], and many students transfer into engineering ( $30 /$ year) during their undergraduate degree. There is an overall lack of efficiency to the university and significant cost of both time and money to the students who transfer into and out of engineering, and it could be avoided if students initially selected the appropriate program. The focus of this study is to examine these two phenomena of entry to engineering programs straight from high school, and from other university undergraduate programs. This study is a work in progress, and as such, this paper will discuss trends emerging from the preliminary data analysis.

\subsection{Purpose of Research}

The purpose of this study is to investigate the factors which affect program choice and govern the changing perspectives and motivations of students who initially choose engineering, and those transfer into engineering programs during their undergraduate degree. The research question driving this study is: "Why do students choose to enter engineering, and what relationship does this entry selection process have with program satisfaction?"

This research intends to fill gaps in the literature, choosing a Canadian, engineering-exclusive perspective. From a long-term perspective, this study as a part of a larger research program that is endeavouring to understand the reasoning for and satisfaction with students' choice to study engineering. These parallel projects are intended to inform a variety of student information sources such as teachers, guidance counsellors and outreach programming, allowing them to better target and inform potential engineering candidates. This ideally will lead to greater educational efficiency and student satisfaction.

\subsection{Literature Review}

The most common application for the examination of student attributes in STEM fields is to relate them to attrition rates. A seminal study from 1991 by Astin and Astin [1] found that in the 388 universities studied, $51 \%$ of engineering students left the program during their undergraduate degree. This study also found that having an engineer or scientist as a father, and peer interest in engineering had a significantly positive correlation with engineering entry. Similar works followed, including Besterfield-Sacre, 1997 [3], which 
sought to model this attrition in engineering programs, in order to identify those most likely to leave and provide them with 'targeted advising and mentoring sessions' to reduce attrition overall.

A 2010 study done by the National Bureau of Economic Research (Arcidiacono, Hotz, \& Kang, 2010 [4]) examined the undergraduate major choices made by Duke University students, based on their perceived ability to pursue alternative career paths, as well as their financial expectations. The study forecasts that if students' financial expectations were corrected to realistic figures, $7.5 \%$ would switch majors. Matusovich, Streveler, \& Miller, 2010 [5], identified traits and values which indicated that a student would complete their engineering degree. The researchers used qualitative techniques, although later coded this data into a low-medium-high scale of 'persistent-related values', 'Attainment', 'Cost', 'Interest' and 'Utility'. They found that those who identified closely with being an engineer were less likely to transfer or drop out. This study was limited by the fact that the school being studied only had engineering programs, which makes faculty transfer impossible without institutional transfer.

\section{STUDY DESIGN}

This study was designed in conjunction with two other research projects at Queen's University, each with the purpose of exploring students' reasoning for choosing engineering. To diversify the information gathered, and broaden the collective scope, each researcher focused on a different demographic, and tuned their study to examine the issues important in that given population. One of these studies examines the use of career resources with Grade 11 and 12 students (the latter half of secondary school), and the other is researching the perspectives of Grade 9 and 10 (the first half of secondary school) students on engineering.

This study uses a third perspective of students already in engineering. This population has distinctive attributes:

- It is easily accessible for study, allowing a researcher to capture a relatively large amount of data with a given amount of resources

- Actual entry decision is known, allowing the research to isolate perspective of those who chose engineering

- Participants have the ability to reflect on their entry decision

These three strengths of the population directly informed the study design.

\subsection{Methodology}

This research follows a qualitative Phenomenological framework, which "aims at gaining a deeper understanding of the nature and meaning of our everyday experiences" [6]. This study consisted of an online survey, followed by in-depth interviews of a smaller number of participants. Preliminary analysis of the survey in Part A was used to indicate points of interest or contention, which allowed for deeper exploration in Part B. The survey data also provide the interviews (Part B) with context. These semi-structured interviews were based on a predetermined set of questions, established during ethics approval, but allowed the interviewer a degree of freedom to explore complex responses more thoroughly. Importantly, these two Parts lend credibility to each other, through a process called triangulation. The theory of triangulation asserts that by extracting data from multiple observers, methods and sources, reliability can be increased [6].

\subsection{Survey Design (Part A)}

The surveys were designed with three guiding principles in mind:

- Questions must identify participant demographics and correspond to research questions

- The survey must attract as many participants, from as many demographics as possible

- The survey must comply with all ethical guidelines

The survey requires participants to enter their year of study, discipline, gender, and whether or not they are an international student, to allow comparison by demographic. The remainder of the survey elicits responses on participants' knowledge of the engineering program and profession, their satisfaction with the engineering program, and the factors involved with their decision.

To maximize participation, reduce redundant contacts, and get a variety of demographics, the researcher personally entered engineering classrooms to describe and pitch the study. For ease of implementation and accessibility, the survey was hosted online through the FluidSurveys platform, and the students were directed to enter a link into their laptop or mobile device, which lead them to the survey host. The last question of the survey asked each participant if they were willing to be interviewed for Part B of the study.

\subsection{Interview Design (Part B)}

Interviews were scheduled with consenting Part A participants, who were selected to maximize variation between participants. This process provides the study with a broader understanding of survey feedback; what experiences prompted those answers, and how important a given answer is to a participant. The semi-structured format of the interview allowed the researcher to delve deeper into the participants' responses, allowing participants the chance to direct the data gathering toward what was important or unique about their situation. 
Participants were selected for interview based on their answers in the Part A survey, ensuring a wide variety of interviewees, based on gender and year. Students who transferred into the engineering program were especially recruited because of their unique perspectives on both engineering and arts/science programs, and their more recent choice of engineering.

\section{ANALYSIS}

Answers from Part A have been partially analyzed using post-hoc descriptive statistics, exploring the data for trends. Further analytical procedures are being investigated. Findings from Part A will be applied to the analysis of Part B. Recordings of interviews were personally transcribed by the interviewer, and will be analyzed with an open-coding process. NVivo software will be used to aid in the coding process.

\section{RESEARCH FINDINGS}

Research findings are currently only available for Part A. There was a $40-80 \%$ survey response rate, varying by class, with $49 \%$ of students using mobile devices or tablets and $51 \%$ using computers. Overall, 416 surveys were received. This data from Part A have been partially analyzed using post-hoc descriptive statistics, exploring the data for trends.

Survey results indicate that the vast majority of students surveyed are satisfied with their choice of engineering as their program of study. $52.5 \%$ of students indicated that they are Very Satisfied; 37.3\%, Satisfied; $3.6 \%$ Unsatisfied; and 6.5\%, Very Unsatisfied. For deeper inspection, the population was divided by gender. For female samples, $\mathrm{n}=165$, and for male samples, $\mathrm{n}=246$.

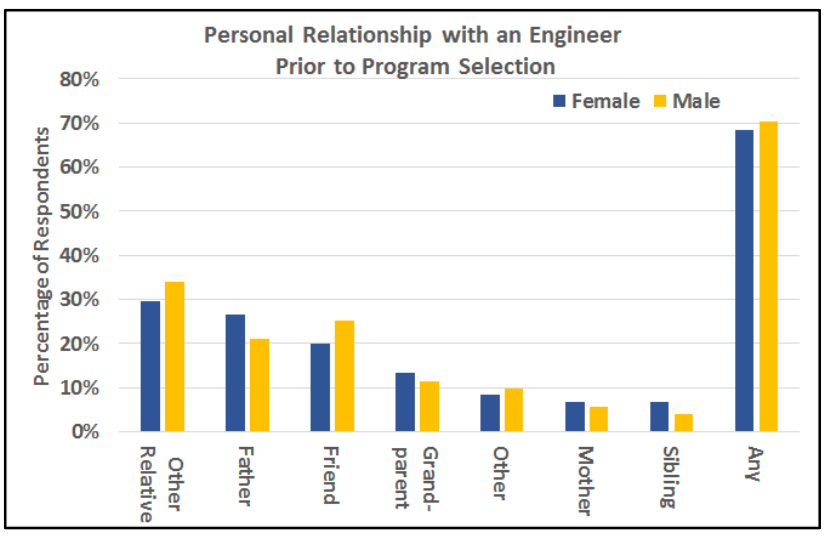

Figure 1: Program Satisfaction of Queen's University engineering undergraduates, as collected in Part A. Participants distinguished by gender.

As is indicated in Figure 1, only small differences were found between female and male populations. $90.1 \%$ of males and $89.7 \%$ of female were indicated they were either "Satisfied" or "Very Satisfied". The largest difference is that $8.6 \%$ of females and $5.3 \%$ of males indicated they were "Very Unsatisfied". Sample sizes were: 2 nd year, $n=206$; 3rd year, $n=102$; 4th year, $n=104$.

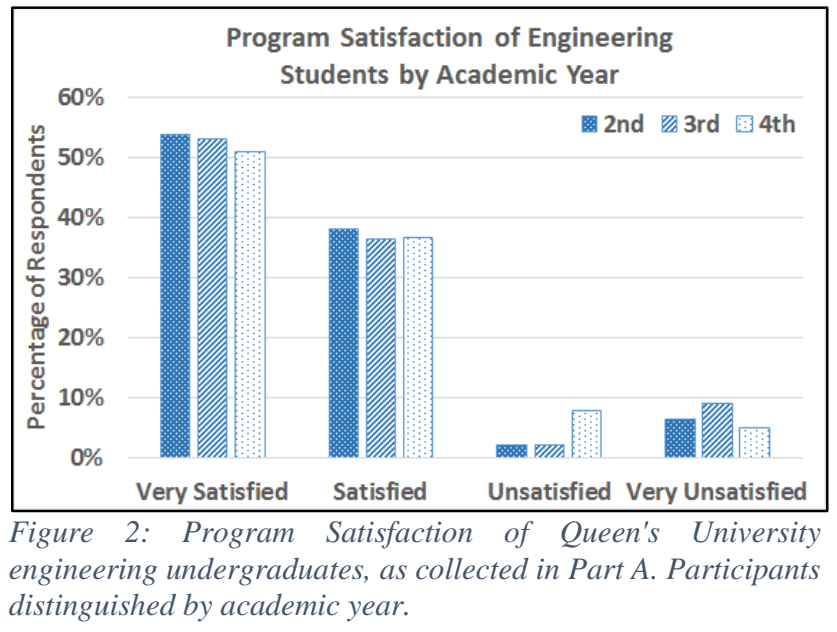

In Figure 2, responses were broken down by academic year. The data is very consistent by year, which suggests that students' satisfaction with the engineering program stays relatively constant over time.

Students were asked to identify the most important factors in their program selection process. The results of this query can be found below in Figure 3.

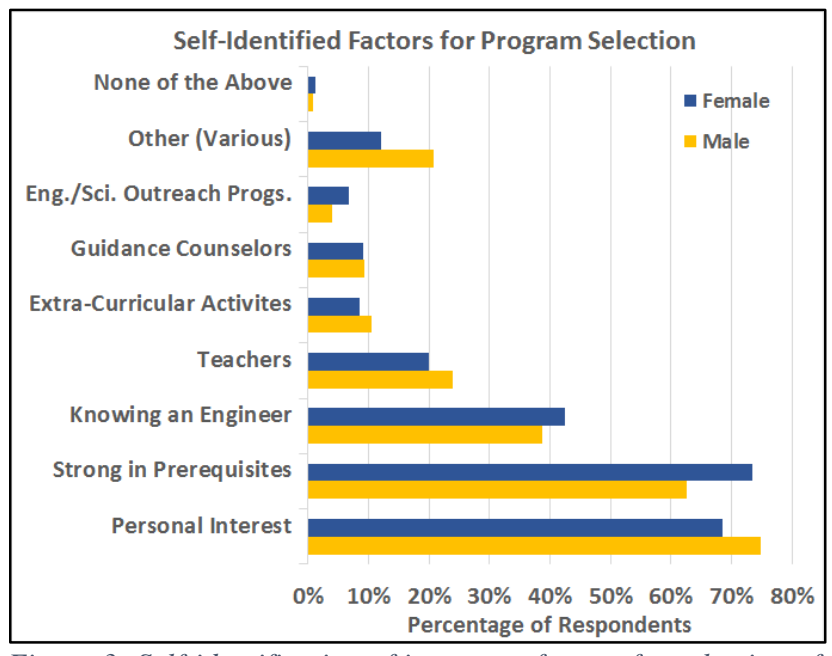

Figure 3: Self-identification of important factors for selection of the engineering program at Queen's University. Participants distinguished by gender.

The two most common factors were "Personal Interest" and "Strength in Prerequisites", and the results were consistent between female and male groups. The two largest gender differentiations were the greater frequency of responses from females of "Engineering/Science Outreach Programs", and "Strength in Prerequisites". 
This study looked to explore the prevalence of personal influence in the process of program selection. Participants were directed to indicate any professional engineers they knew personally, when they made their initial program selection. The "Any" column was generated by accumulating students who selected one or more of the available choices. Results are displayed below in Figure 4.

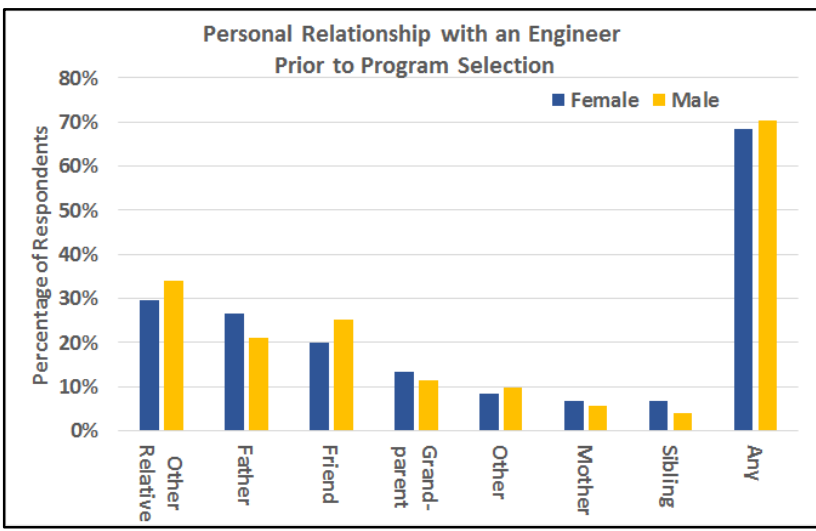

Figure 4: Personal relationships of Queen's engineering undergraduate students with professional engineers, prior to program selection. Participants distinguished by gender.

There data was fairly consistent by gender. Only small differentiations occurred, although one pattern is indicated. Direct family relations of "Mother", "Father", "Grandparent", and "Sibling" were more commonly indicated by female participants, while "Other Relative", "Friend" and "Other" were more commonly indicated by males.

The proportion of students who qualified for the "Any" column (69.7\%) was higher than expected. With no control sample, it is difficult to draw conclusive meaning from this, but it merits further study.

\section{CONCLUSIONS AND NEXT STEPS}

The various results from this preliminary investigation will be valuable looking forward to the Part $\mathrm{B}$ analyses. The most prominent finding was that there was a high level of consistency in gender and year. Males and females indicated that a similar set of factors were important in their program selection, and both genders rated their satisfaction with their program choice highly. Overall, $90 \%$ of students indicated satisfaction with their choice of engineering. The data agreed with the literature in that a large proportion of students $(23.1 \%)$ have a father who is a professional engineer (from Astin and Astin [1]). This study also indicated that $69.7 \%$ of students personally knew an engineer, in some capacity, prior to their application to an engineering program.

The next step for this project is to analyze the interview data from Part B. This analysis should provide a much deeper understanding of the phenomena, and explore the trends found in Part A more closely. After completion of this study, its value could be significantly increased by surveying at more universities. Currently the data is all from one medium-size Ontario university, and collecting data from additional universities would increase the strength of the study, as well as allowing for comparison between provinces, regions and schools.

\section{Acknowledgements}

This paper, and the larger research study, would not have been possible without funding from NSERC through the Chairs in Design Engineering program, support from the community of engineering education researchers at Queen's, and the participation of the students who volunteered their time for this study.

\section{References}

[1] A. W. A. a. H. S. Astin, "Undergraduate Science Education: The Impact of Different College Environments on the Educational Pipeline in the Sciences," University of California, Higher Education Research Institute, Los Angeles, 1992.

[2] CUDO: Common University Data Ontario, "Key Performance Indicators, Degree Completion Rate for Undergraduate Cohort, New Year 1 Students," [Online]. Available: http://cudo.cou.on.ca/. [Accessed 2 May 2015].

[3] M. Besterfield-Sacre, C. J. Atman and L. J. Shuman, "Characteristics of Freshman Engineering Students: Models for Determining Studnet Attrition in Engineering," Journal of Engineering Education, pp. 139-149, 1997.

[4] P. Arcidiacono, V. J. Hotz and S. Kang, "Modeling Colled Major Choices Using Elicited Measure of Expectations and Counterfactuals," National Bureau of Economic Research, Cambridge, MA, 2010.

[5] H. M. Matusovich, R. A. Streveler and R. L. Miller, "Why Do Students Choose Engineering? A Qualitative, Longitudinal Investigation of Students' Motivational Values," Journal of Engineering Education, pp. 289-303, 2010.

[6] M. Q. Patton, Qualitative Research \& Evaluation Methods, 3rd ed., Thousand Oaks: Sage Publications, 2002. 